\title{
The Effects of Type of Surgery and Time on Psychological Adjustment in Women After Breast Cancer Treatment
}

\author{
Lorenzo Cohen, PhD, Thomas F. Hack, PhD, Carl de Moor, PhD, \\ Joel Katz, PhD, and Paul E. Goss, MD, PhD
}

\begin{abstract}
Background: The aim of the present study was to examine whether type of surgery, age, and time since surgery influenced psychological distress and quality of life (QOL) in women treated for breast cancer.

Methods: We surveyed 183 women who had undergone surgery for breast cancer. Psychological distress was measured with the Mental Health Inventory and QOL was measured with the European Organization for Research and Treatment of Cancer Quality of Life Questionnaire.

Results: After controlling for stage of disease, radiation treatment, and age, there was a statistically significant interaction between type of surgery and time since surgery for the Mental Health Inventory total score, and a marginal interaction between type of surgery and time since surgery for the Global health status/QOL score. Women who had breast conservation surgery experienced significantly greater levels of psychological distress and marginally worse QOL from 40 months after surgery onward than did women who received a mastectomy.

Conclusions: The effects of different surgical treatments for breast cancer on psychological distress and QOL become apparent only after a period of several years. Women, therefore, need counseling on the potentially positive and negative psychological implications of different surgical treatments for breast cancer.
\end{abstract}

Key Words: Breast cancer-Quality of life-Mental health-Surgery.

Women diagnosed with breast cancer must make many difficult treatment decisions. They often have a choice between modified radical mastectomy (mastectomy) or partial mastectomy called breast conservation surgery (BCS), which is usually followed by irradiation. Women may also have the option of having a mastectomy followed by immediate reconstruction of the breast. If lymph nodes in the axilla are histologically involved, either surgery option is typically followed by chemother-

Received February 2, 2000; accepted April 5, 2000

From the Department of Behavioral Science, The University of Texas M. D. Anderson Cancer Center, Houston, Texas (LC, CdM); the Faculty of Nursing, University of Manitoba, Winnipeg, Manitoba, Canada (TFH); The Toronto Hospital and University of Toronto, Toronto, Ontario, Canada (JK, PEG); and Princess Margaret Hospital, Toronto, Ontario, Canada (PEG).

Presented in part at The Society of Behavioral Medicine, 21 st Annual Scientific Sessions, Nashville, Tennessee, April 5-8, 2000

Address correspondence and reprint requests to: Lorenzo Cohen, $\mathrm{PhD}$, Department of Behavioral Science, The University of Texas M. D. Anderson Cancer Center, 1515 Holcombe Boulevard, Box 243, Houston, TX 77030; Fax: 713-745-4286; E-mail: Icohen@, notes.mdacc.tmc.edu apy. The 5-year survival and disease-free survival rates are comparable for women with similar disease characteristics who undergo either BCS plus radiation therapy or a mastectomy. ${ }^{1-3}$ This makes the treatment decision difficult for women who have the option of BCS, mastectomy, or mastectomy with immediate reconstruction, and psychological adjustment after treatment is a factor to consider.

The effects of different surgical approaches on psychological adjustment and quality of life (QOL) have been extensively examined. ${ }^{4,5}$ In addition, a recent metaanalysis of 40 investigations examined postsurgical adjustment in women who underwent BCS or mastectomy. After controlling for unpublished negative findings, body/self-image was the only factor that significantly differed between the treatment groups, with women who underwent BCS reporting better body/self-image. ${ }^{6}$ In contrast, two studies suggest that women who undergo a mastectomy may have better psychological adjustment than women who undergo BCS. ${ }^{7,8}$ In addition, Levy et al. ${ }^{9}$ found that at 15 months postsurgery, women who 
underwent a mastectomy reported greater levels of emotional support than women who had BCS, but the differences in mood were no longer significant.

The research on adjustment and QOL of women with breast cancer is consistent in showing that there are few differences between which type of surgical treatment is chosen. However, age is a factor that has been observed to affect psychological outcomes, with older women reporting better psychological adjustment than younger women.5,10-13 In addition, QOL and psychological adjustment steadily improve over time for all age groups. Most of this research, however, assessed adjustment in only the early months or within the first several years after treatment. It is conceivable that differences in adjustment emerge over time and adjustment might also vary by age. This would be important information for patients making treatment decisions.

There is some evidence that BCS offers more psychological "protection" for younger women. ${ }^{13-16}$ For example, a recent study in which women were randomly assigned to surgical treatment found that younger women who had a mastectomy reported worse body image than did the women who had BCS. ${ }^{14}$ Similarly, a study examining the effects of age, type of surgery, and time since surgery on psychological distress in long-term breast cancer survivors revealed that age was an important factor and there was an interaction between age and type of surgery. ${ }^{15}$ In particular, younger women $(<50$ years) who underwent a mastectomy had the highest levels of psychological distress, older women $(\geq 50$ years) who underwent a mastectomy had the lowest levels of psychological distress, and women who received BCS had intermediate distress scores that were equivalent across the age groups. These findings suggest that for younger women, BCS was protective against the distress associated with a mastectomy and for older women, BCS was associated with higher distress than was a mastectomy.

The aim of the present study was to further examine whether the type of surgery, age, and time since surgery influence psychological adjustment and QOL in women treated for breast cancer. This is the secondary aim of a cross-sectional study designed to assess physical and psychological morbidity specifically associated with axillary lymph node dissection (ALND) for breast cancer. ${ }^{10}$ We hypothesized that the effects of surgery on psychological adjustment and QOL would only be apparent when the interaction among type of surgery, age, and time since surgery were examined.

\section{PATIENTS AND METHODS}

\section{Study Population}

A consecutive sample of patients was recruited from the breast medical oncology clinics at The Toronto Hospital and Princess Margaret Hospital, Toronto, Canada, between March 1995 and February 1997. Patients were eligible for the study if they had undergone breast cancer surgery with ALND and if they fulfilled the following seven criteria: (1) at least 6 months had elapsed since surgery or chemotherapy or radiation treatment (to eliminate the acute effects of treatment on the outcome measures) and no more than 5 years had elapsed since surgery (the time when patients are typically viewed as "cancer free"); (2) there was no distant metastasis at the time of the study; (3) there was no local recurrence at the time of the study; (4) there was no evidence of psychiatric history or a current psychiatric disorder, dementia, or chronic pain that predated surgery; (5) the patient was 18 years of age or older; (6) the patient was able to read and speak English; and (7) the patient was female.

\section{Procedures}

A detailed description of the procedures and measures used in this study has been published. ${ }^{10}$ Briefly, patients were initially mailed an invitation to participate in the study. They were then recruited at a routine follow-up clinic visit. Patients who expressed an interest in participating were given a full summary of the study at the clinic visit. Any patient questions were answered, and consent was obtained. Participants then underwent a physical therapy assessment to assess arm/shoulder range of motion and were asked to complete several questionnaires. The study was approved by The Toronto Hospital Committee for Research on Human Subjects.

\section{Measures}

A subset of the measures used in the larger study ${ }^{10}$ were used in the present study. The main variables of interest were those related to psychological distress and QOL and are described in detail below. Treatment variables, disease characteristics, and patient background variables were abstracted from patients' charts.

\section{Mental Health Inventory}

The Mental Health Inventory (MHI) ${ }^{17}$ measures symptoms of psychological distress along five dimensionsanxiety, depression, loss of behavioral/emotional control, positive affect, and interpersonal ties - and also yields a total score. ${ }^{18}$ For the regression analyses, we used the total score (MHI-Total) as the main measure of psychological distress to decrease the overall number of analy- 
ses. The reliability of the total score was $r=.93$ as measured by Cronbach's alpha, with high scores representing worse psychological distress.

\section{QOL Questionnaire}

The European Organization for Research and Treatment of Cancer Quality of Life Questionnaire (EORTC QLQ-C30) ${ }^{19}$ is an internationally recognized instrument for assessing QOL in cancer patients. It is composed of five functional scales (physical, role, cognitive, emotional, and social); three symptom scales (fatigue, pain, and nausea and vomiting); six single items that measure dyspnea, sleep quality, appetite, constipation, diarrhea, and financial impact; a global QOL item; and a global health status item. The questionnaire is psychometrically sound $^{20,21}$ and has been used to identify meaningful changes in QOL over time. ${ }^{22}$ For an overall measure of QOL for the regression analyses, we used the Global health status/QOL score which is a combination of the global QOL item and the global health status item. Low scores on this scale are predictive of poor survival. ${ }^{23,24}$ The Cronbach's alpha for this scale was .80 in this sample of patients. The Global health status/QOL scale was significantly correlated with all the functional and symptom scales (all $P$ values were $<.0001$ ) and ranged from .29 (social) to .61 (fatigue).

\section{Statistical Analysis}

Onc-way analyses of variance and the $\chi^{2}$ test for categorical variables were conducted to identify any differences between the type of surgery and demographic and medical variables, psychological distress, and QOL. Multiple regression analyses were then performed separately for the MHI-Total and the Global health status/ QOL scales to determine whether psychological distress and QOL were associated with age, type of surgery, or time since surgery. Subsequent analyses examined the three two-way interaction terms (age by type of surgery, age by time since surgery, and type of surgery by time since surgery), and the three-way interaction term (age by type of surgery by time since surgery) with age, type of surgery, and time since surgery entered first, followed by the interaction terms. All regression analyses were conducted adjusting for stage of disease and radiation treatment.

\section{RESULTS}

Two hundred forty-eight women were approached to participate in the main study. Of these, 18 declined and 6 were not included in the analyses because they had not undergone an ALND. In 183 of the 224 women who participated in the main study, at least 6 months had clapsed since surgery or adjuvant treatment and no more than 5 years had elapsed since surgery. Thirty-nine patients were excluded from the analyses because more than 5 years had elapsed since surgery. There were no significant demographic or medical characteristic differences between these women and the rest of the sample. Sixty-seven percent ( $n=123$ ) of the sample had had $\mathrm{BCS}$, and $33 \%(\mathrm{n}=60)$ had had a modified radical mastectomy. None of the patients had received breast reconstruction surgery. The mean age of the entire sample was $57.1 \pm 11.7$ (range: $30-79$ years of age) and only $27 \%$ of the population was under 50 years of age. The medical characteristics of the sample are presented in Table 1.

The $\chi^{2}$ tests comparing the type of surgery (BCS vs. mastectomy) and stage of disease, the number of positive regional nodes, tumor size, and adjuvant treatment showed that a significantly greater number of women who underwent a mastectomy had stage III disease and tumors $>5 \mathrm{~cm}$ in diameter (Table 1). Not surprisingly, more women who had BCS also received radiation treatment. There were no group differences in either the number of positive regional nodes or in the number of women who received chemotherapy and tamoxifen treatment. One-way analyses of variance examining the type of surgery in association with other demographic and medical characteristics, psychological distress, and QOL revealed few differences. Women who underwent BCS had significantly smaller tumors than did women who underwent a mastectomy $[2.0 \pm 1.2 \mathrm{~cm}$ vs. $2.7 \pm 2.2 \mathrm{~cm}$; $F(1,181)=6.64 ; P<.01]$. There were no differences between the women who underwent BCS and mastectomy on age $(56.9 \pm 11.4$ vs. $57.5 \pm 12.5)$, months since diagnosis $(24.1 \pm 12.0$ vs. $26.5 \pm 14.0)$, or number of regional nodes positive $(1.4 \pm 3.5$ vs. $1.3 \pm 3.1)$. As can be seen in Table 2, women who underwent BCS reported better QOL on the social functioning scale of the EORTC QLQ-C30 than did women who underwent a mastectomy. However, there were no significant differences between the type of surgery and MHI scores or the other scales of the EORTC QLQ-C30.

\section{Psychological Distress}

After entering age, the type of surgery, stage of disease, radiation treatment, and time since surgery into one equation, we found that only age was associated with MHI-Total score $(F=4.75 ; \beta=-.17, P<.03)$. The negative association between age and the MHI-Total score indicated that younger women reported worse psychological distress. However, regression analyses of the two-way and three-way interactions between age, time 
TABLE 1. Medical characteristics

\begin{tabular}{lcccc}
\hline & $\begin{array}{c}\text { Breast conservation } \\
\text { surgery }(\%) \\
(\mathrm{n}=123)\end{array}$ & $\begin{array}{c}\text { Mastectomy } \\
(\%)(\mathrm{n}=60)\end{array}$ & $\chi^{2}$ & $P$ value \\
\hline $\begin{array}{l}\text { Number of regional nodes positive } \\
0\end{array}$ & 65 & 60 & & \\
1 & 17 & 22 & & \\
$2-5$ & 11 & 12 & & .88 \\
$\geq 6$ & 7 & 7 & .67 & \\
Tumor size & 65 & 55 & & \\
$\leq 2 \mathrm{~cm}$ & 34 & 28 & & \\
$>2$ and $\leq 5 \mathrm{~cm}$ & $2^{\mathrm{a}}$ & $17^{\mathrm{a}}$ & 13.61 & .001 \\
$>5 \mathrm{~cm}$ & 43 & 39 & & \\
Stage of disease & & & \\
$\quad$ I & 55 & 48 & & .01 \\
II & $2^{\mathrm{a}}$ & $13^{\mathrm{a}}$ & 9.29 & .0001 \\
III & & & & \\
Adjuvant treatment & 85 & 13 & 86.64 & .11 \\
Radiation treatment & 33 & 36 & .21 & .65 \\
Chemotherapy treatment & 56 & 54 & & \\
Tamoxifen & & & & \\
\hline
\end{tabular}

a Values differ significantly from each other.

${ }^{\mathrm{b}}$ Stage was calculated using the tumor, node, metastasis classification system.

since surgery, and type of surgery on MHI-Total score revealed a significant interaction only between time since surgery and type of surgery $(F=5.90 ; \beta=-.68$; $P<.02)$. Figure 1 represents the interaction between the time since surgery and $\mathrm{MHI}-$ Total scores separately for the mastectomy and BCS groups. The lines were computed using the fitted regression model coefficients and represent the predicted MHI-Total scores for each surgery group, adjusting for the stage of disease, radiation treatment, and age. The slopes of the lines suggest that women who underwent BCS experienced an increase in psychological distress over time, whereas women who received a mastectomy experienced a decrease in psychological distress over time. Importantly, the lines cross at approximately 20 months after surgery, suggesting that women who underwent BCS experienced lower levels of psychological distress during the first 20 months after surgery but greater levels of distress afterward.

To determine the points in time that the two groups are significantly different, we plotted the difference in the predicted MHI-Total score between the BCS and mastectomy groups (Fig. 2). This line was computed simply

TABLE 2. EORTC QLQ-C3O and MHI scores among women who received breast conservation surgery and mastectomy

\begin{tabular}{|c|c|c|c|c|}
\hline & $\begin{array}{c}\text { Breast conservation } \\
\text { surgery (Mean } \pm \mathrm{SD}) \\
(\mathrm{n}=123)\end{array}$ & $\begin{array}{c}\text { Mastectomy } \\
(\text { Mean } \pm S D) \\
(\mathrm{n}=60)\end{array}$ & $F$ & $P$ value \\
\hline \multicolumn{5}{|l|}{ Scores on $\mathrm{MHI}^{\mathrm{a}}$} \\
\hline Depression & $21.38 \pm 18.03$ & $21.25 \pm 21.80$ & .00 & .97 \\
\hline Anxiety & $27.18 \pm 18.80$ & $23.80 \pm 18.74$ & 1.31 & .25 \\
\hline Behavioral control & $14.18 \pm 13.63$ & $16.75 \pm 18.36$ & 1.13 & .29 \\
\hline Positive affect & $30.65 \pm 18.55$ & $27.83 \pm 21.15$ & .88 & .35 \\
\hline Total & $23.26 \pm 13.53$ & $22.53 \pm 15.63$ & .08 & .77 \\
\hline \multicolumn{5}{|l|}{ Scores on EORTC QLQ-C $30^{\mathrm{b}}$} \\
\hline Physical functioning & $83.09 \pm 19.34$ & $84.33 \pm 14.78$ & .19 & .66 \\
\hline Role functioning & $85.77 \pm 26.02$ & $85.00 \pm 24.86$ & .04 & .85 \\
\hline Emotional functioning & $75.74 \pm 21.77$ & $76.80 \pm 23.12$ & .09 & .76 \\
\hline Cognitive functioning & $83.74 \pm 21.28$ & $83.61 \pm 19.77$ & .00 & .96 \\
\hline Social functioning & $92.54 \pm 12.19$ & $87.50 \pm 19.32$ & 3.99 & .05 \\
\hline Fatigue symptoms & $22.22 \pm 20.27$ & $22.78 \pm 22.91$ & .03 & .87 \\
\hline Nausea and vomiting symptoms & $3.82 \pm 9.53$ & $3.61 \pm 8.73$ & .02 & .88 \\
\hline Pain symptoms & $12.43 \pm 16.53$ & $15.28 \pm 21.10$ & .99 & .32 \\
\hline Global health status/QOL & $74.52 \pm 17.21$ & $75.27 \pm 19.59$ & .07 & .79 \\
\hline
\end{tabular}

${ }^{a}$ Standard scores $(0-100)$ are presented for each scale and total score of the MHI. Higher scores indicate worse mental health.

${ }^{\mathrm{b}}$ Standard scores $(0-100)$ are presented for each scale of the EORTC QLQ-C30. Higher scores for the functional and Global health status/QOL scales indicate better quality of life, and higher scores for the symptom scales indicate worse quality of life. 


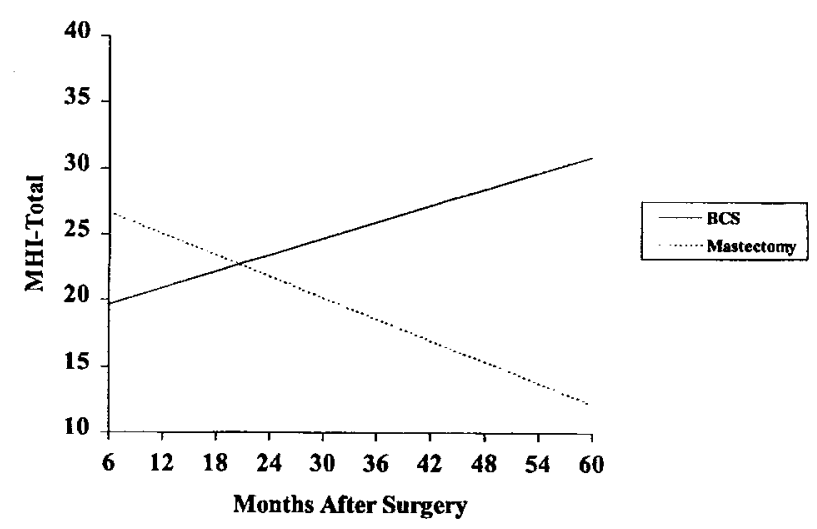

FIG. 1. Interaction between type of surgery and time since surgery for standardized MHI-Total scores, adjusting for stage of disease, radiation treatment, and age. Higher scores indicate worse mental health.

by subtracting the predicted MHI-Total scores of the mastectomy group from those of the BCS group. A $95 \%$ confidence band around the line, represented by the outer lines, was computed using standard linear regression procedures. Points in time since surgery where the confidence band does not include zero represent periods during which the differences between the two groups were statistically significant. The lower bound of the confidence band approaches but does not exceed zero at the earliest time since surgery, indicating only a marginally significant difference in the MHI-Total scores between the two groups of patients at this time. However, the upper bound of the confidence band drops below zero at approximately 40 months after surgery, indicating that women who underwent BCS experienced significantly greater levels of psychological distress from this point onward than did women who underwent a mastectomy.

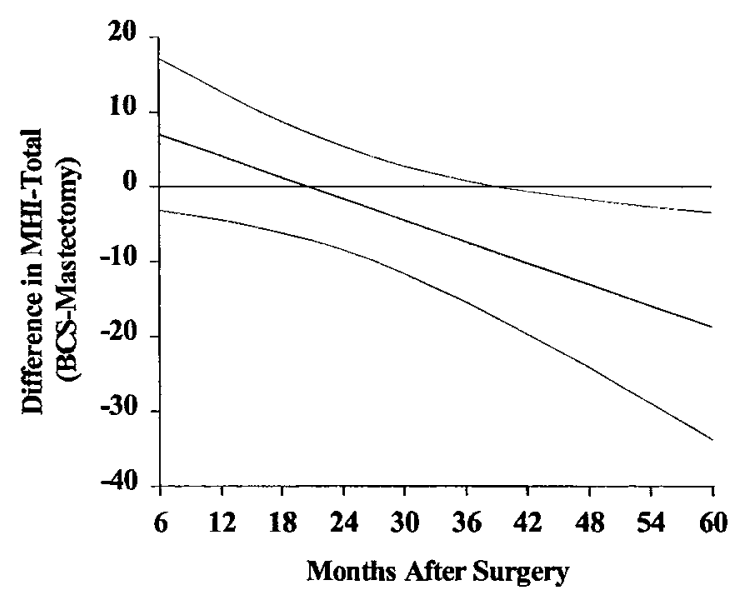

FIG. 2. Difference plus the $95 \%$ confidence band in predicted standardized MHI-Total scores between patients who had a mastectomy and those who had BCS.

\section{Quality of Life}

Examination of the effects of age, time since surgery, and type of surgery on Global health status/QOL, after controlling for the stage of disease and radiation treatment, revealed that only the time since surgery was positively associated with Global health status/QOL ( $F$ $=6.81 ; \beta=0.20 ; P<.01)$. This suggests that there was an overall increase in Global health status/QOL over time. Age and type of surgery were not associated with Global health status/QOL. Regression analyses of the two-way and three-way interactions between age, time since surgery, and type of surgery on Global health status/QOL revealed only a trend for the interaction between time since surgery and type of surgery $(F=$ $2.56 ; \beta=0.45 ; P<.11$ ). Similar to the results for psychological distress, the data suggest that women who underwent $\mathrm{BCS}$ experienced little change in QOL over time, whereas women who underwent a mastectomy experienced marginally better QOL over time.

\section{DISCUSSION}

We found few differences in QOL and psychological distress between women who underwent BCS and those who underwent a mastectomy. Importantly, differences did become apparent when we examined changes in psychological distress over time. Overall, age was also negatively associated with psychological distress, and time since surgery was associated with better QOL. As was expected, the two groups were also different from the standpoint of the stage of disease and adjuvant treatment administered, with more women who underwent a mastectomy having stage III disease (attributable to their having larger tumors) and more women who had BCS receiving radiation treatment.

Previous research has reported few differences between the two types of surgery, and those differences reported were associated with better body/self-image for women who received BCS. ${ }^{4,6,14}$ Although the difference in social functioning as measured by the EORTC QLQC30 was statistically significant, both surgery groups reported levels of social functioning higher than the levels of all other scales on the EORTC QLQ-C30. The EORTC QLQ-C30 does not have a direct measure that assesses body/self-image, but the differences in social function measured by this instrument might reflect differences associated with body/self-image.

We found that age was negatively associated with psychological distress, which is consistent with the findings for our full database of 222 patients ${ }^{10}$ and findings from other studies. ${ }^{5.11-13.15}$ This suggests that younger women have greater difficulty adjusting after breast can- 
cer treatment than do older women. Not surprisingly, QOL was also positively associated with the time since surgery, suggesting that QOL improves over time for all women.

Differences between women who underwent BCS vs. mastectomy became apparent when we examined changes in psychological distress over time. After controlling for stage of disease, radiation treatment, and age, we found that women who underwent a mastectomy reported marginally worse psychological distress 6 months after surgery but significantly less psychological distress from 40 months after surgery onward than did women who underwent BCS. This finding is consistent with the studies by Fallowfield et al. ${ }^{7}$ and Levy et al., ${ }^{8}$ which reported worse psychological adjustment in women who received BCS than in women who received a mastectomy.

There was no interaction between age and type of surgery or between age and time since surgery for psychological distress. Although we found a main effect for age, suggesting that older women reported less psychological distress, the lack of an interaction between age and type of surgery suggests that differences between the two surgery groups in psychological distress and QOL did not vary according to age. This is inconsistent, however, with the study by Dorval et al., ${ }^{15}$ which reported an interaction between age and type of surgery. These researchers found that young women ( $<50$ years) who underwent a mastectomy reported worse psychological distress than did older women ( $\geq 50$ years) who underwent a mastectomy. One possible explanation for the lack of an interaction between age and type of surgery in our study is that our patient population was older than that in the Dorval et al. ${ }^{15}$ study. Only $27 \%$ of our patients were younger than 50 , whereas almost $50 \%$ of the patients in the study of Dorval et al. ${ }^{15}$ were younger than 50 . With fewer than 15 women under 50 years old who underwent a mastectomy in our patient population, the present study may be underpowered to find an interaction between age and type of surgery. Our findings are, therefore, somewhat consistent with those of Dorval et al. ${ }^{15}$ in that the majority of our sample was more than 50 years old and, for women more than 50 years old, BCS was associated with higher levels of psychological distress. ${ }^{15}$

If validated, the significant differences in psychological distress and potential differences in QOL in the two surgery groups from 40 months onward that were noted in our study have important implications for surgical treatment decisions. Although women who undergo BCS as a whole tend to report better body/self-image than do women who undergo mastectomy, ${ }^{4,6,14}$ our data suggest that women who undergo BCS go on to report significantly worse levels of psychological distress 40 months after surgery and onward than do women who undergo mastectomy. It is important to note, however, that the overall level of psychological distress was relatively low, and QOL scores were quite high in this population. This is consistent with findings from research comparing breast cancer survivors with age-matched controls. ${ }^{12.25}$ However, there was as much as an $18 \%$ difference in the predicted mental health scores between women who underwent a mastectomy and those who underwent BCS at approximately 60 months after surgery. This great a difference in mental health scores suggests there might be a clinically significant difference in mental health between the two groups that emerges over time.

It is not clear why women who had BCS reported worse adjustment than the women who had mastectomies. One explanation might be that significantly more women who underwent BCS also had radiation treatment, and the possible long-term side effects of radiation treatment may contribute to the group differences. However, we controlled for radiation treatment in the analyses, and radiation treatment was not directly associated with psychological distress nor QOL. Similarly, women who underwent a mastectomy had more advanced disease and a worse prognosis. Therefore, they may be expected to have improved mental health over time as they become more confident in remaining disease free. Although plausible, we did control for stage of disease in all analyses, and stage of disease also was not independently associated with psychological distress or QOL. Alternatively, because women who had BCS still have an intact breast, they might have increased anxiety about disease recurrence. We did not, however, collect any data that could assess this possibility. Further research is therefore needed to explore the association between surgical technique and concerns about disease recurrence over an extended period of time as a predictor of psychological distress and QOL.

There are a number of limitations to our study that need consideration. For instance, the cross-sectional nature of this study makes it difficult to know whether the surgery by time effect could be caused by pretreatment differences in the two surgery groups. On the other hand, the cross-sectional nature of the data provides more data points across time since surgery, which is more difficult to obtain with prospective data collection. Another limitation is that it was not possible to randomize patients to the surgical procedures, so there might be a selection bias that could influence long-term adjustment. For example, there might be individual differences between women who elect to have BCS vs. mastectomy that could influ- 
ence adjustment. There is also a potential survival bias in the current study in that women who are alive several years from the time of surgery might be different from those alive at earlier time points. This may be particularly relevant for the data points further out in time. In particular, the women with more advanced disease, who therefore have a lower 5-year survival probability, might represent a biased population in terms of their psychological status and QOL over time. In addition, we did not recruit any women who were $<6$ months from the time of surgery, so it is not clear what differences may exist at this early time point. Finally, as mentioned above, only $27 \%$ of our sample was under 50 years of age. Therefore, the results might not accurately represent how younger women would adjust over time to the different surgical techniques.

Differences in psychological distress and QOL associated with surgical treatments for breast cancer may be different depending on the length of time since surgery. Body/self-image is a significant problem for many breast cancer patients, particularly women who undergo a mastectomy. However, interestingly, in the present study we found that psychological distress was worse in women who underwent BCS than in women who underwent a mastectomy, but only 40 months or more after surgery. Therefore, although disease-free survival and overall survival data are similar for women with small localized disease who undergo either BCS or mastectomy, women need to be counseled on the potential positive and negative psychological effects of different surgical treatments. For example, women who elect to have BCS may have good body/self-image, but having an intact breast may also cause anxiety about disease recurrence. There are still a number of individual difference variables to be examined to know more fully which surgical treatment will result in the best long-term adjustment for an individual woman. This problem may be further mitigated by innovations and improvements in surgical techniques, such as mastectomy with immediate breast reconstruction, that may help a woman maintain body/self-image yet eliminate the possible fear of recurrence associated with BCS. Future research should prospectively explore the QOL effects of different surgical treatments for breast cancer by examining age and changes in psychological adjustment and QOL over time. Findings yielded by such research would help women make more informed treatment decisions.

Acknowledgments: Grant funding provided by the Canadian Breast Cancer Foundation (TFH). We thank Beth Notzon, from the Office of Scientific Publications, The University of
Texas M. D. Anderson Cancer Center, for her helpful editorial comments on this article.

\section{REFERENCES}

1. Fisher B, Bauer M, Margolese R, et al. Five-year results of a randomized clinical trial comparing total mastectomy and segmental mastectomy with or without radiation in the treatment of breast cancer. $N$ Engl J Med 1985;312:665-73.

2. Fisher B, Redmond C, Poisson R, et al. Eight-year results of a randomized clinical trial comparing total mastectomy and lumpectomy with or without irradiation in the treatment of breast cancer. $N$ Engl J Med 1989;320:822-28. (Published erratum appears in $N$ Engl J Med 1994;330(20):1467.)

3. Fisher B, Anderson S, Redmond CK, Wolmark N, Wickerham DL, Cronin WM. Reanalysis and results after 12 years of follow-up in a randomized clinical trial comparing total mastectomy with lumpectomy with or without irradiation in the treatment of breast cancer. $N$ Engl J Med 1995;333:1456-61,

4. Kiebert GM, de Haes JCM, van de Velde CJH. The impact of breast-conserving treatment and mastectomy on the quality of life of early-stage breast cancer patients: a review. J Clin Oncol 1991: 9:1059-70.

5. Moyer A, Salovey P. Psychosocial sequelae of breast cancer and its treatment. Ann Behav Med 1996;18:110-25.

6. Moyer A. Psychosocial outcomes of breast-conservation surgery versus mastectomy: A meta-analytic review. Health Psychol 1997; $16: 284-9$

7. Fallowfield LJ, Baum M, Maguire GP. Effects of breast conservation on psychological morbidity associated with diagnosis and treatment of early breast cancer. $B M J$ 1986;293:1331-4.

8. Levy SM, Herberman RB, Lee JK, Lippman ME, d'Angelo T. Breast conservation versus mastectomy: distress sequelae as a function of choice. J Clin Oncol 1989;7:367-75.

9. Levy SM, Haynes LT, Herberman RB, Lee J, McFeeley S, Kirkwood J. Mastectomy versus breast conservation surgery: mental health effects at long-term follow-up. Health Psychol 1992;11: $349-54$.

10. Hack T, Cohen L, Katz J, Robson L, Goss P. Physical and psychological morbidity following axillary lymph node dissection for breast cancer. J Clin Oncol 1999;17:143-9.

11. Ganz PA, Lee JJ, Sim MS, et al. Exploring the influence of multiple variables on the relationship of age to quality of life in women with breast cancer. J Clin Epidemiol 1992;45:473-85.

12. Ganz PA, Rowland JH, Desmond K, Meyerowitz BE, Wyatt GE. Life after breast cancer: understanding women's health-related quality of life and sexual functioning, J Clin Oncol 1998;16:50I14.

13. McCaul KD, Sandgren AK, King B, O'Donnell S, Branstetter A, Foreman G. Coping and adjustment to breast cancer. Psycho-Oncol 1999;8:230-6.

14. Poulsen B, Graversen HP, Beckmann J, Blichert-Toft M. A comparative study of post-operative psychosocial function in women with primary operable breast cancer randomized to breast conservation therapy or mastectomy. Eur J Surg Oncol 1997;23:327-34.

15. Dorval M, Maunsell E, Deschenes L, Brisson J. Type of mastectomy and quality of life for long term breast carcinoma survivors. Cancer 1998;83:2130-8.

16. Schover LR. Sexuality and body image in younger women with breast cancer. $J$ Natl Cancer Inst 1994;16:177-82.

17. Ware JE, Johnston SA, Davies-Avery A, et al. Conceptualization and Measurement of Health for Adults in the Health Insurance Study (Mental Health R-1987/3-HEW: 3). Santa Monica, CA: RAND Corporation, 1994.

18. Weinstein MC, Berwick DM, Goldman PA, Murphy JM, Barsky AJ. A comparison of three psychiatric screening tests using re- 
ceiver operating characteristic (ROC) analysis. Med Care 1989 . 27:593-607.

19. Aaronson NK, Ahmedzai S, Bergman B, et al. The European Organization for Research and Treatment of Cancer QLQ-C30: a quality-of-life instrument for use in international clinical trials in oncology. J Natl Cancer Inst 1993;85:365-76.

20. Aaronson NK, Bartelink H, van Dongen JA, van Dam FS. Evaluation of breast conserving therapy: clinical, methodological and psychosocial perspectives. Eur J Surg Oncol 1988;14:133-40.

21. Hjermstad MJ, Fossa SD, Bjordal K, Kaasa S. Test/retest study of the European Organization for Research and Treatment of Cancer Core Quality-of-Life Questionnaire. $J$ Clin Oncol 1995;13:1249-54.

22. Osoba D, Rodrigues G, Myles J, Zee B, Pater J. Interpreting the significance of changes in health-related quality-of- life scores. $J$ Clin Oncol 1998;16:139-44.

23. Coates A, Porzsolt F, Osoba D. Quality of life in oncology practice: prognostic value of EORTC QLQ-C30 scores in patients with advanced malignancy. Eur $J$ Cancer 1997;33:1025-30.

24. Dancey J, Zee B, Osoba D, et al. Quality of life scores: an independent prognostic variable in a general population of cancer patients receiving chemotherapy. The National Cancer Institute of Canada Clinical Trials Group. Qual Life Res. 1997:6:151-8.

25. Dorval M, Maunsell E, Deschenes L, Brisson J, Masse B. Long-term quality of life after breast cancer: comparison of 8-year survivors with population controls. $J$ Clin Oncol 1998; $16: 487-94$ 